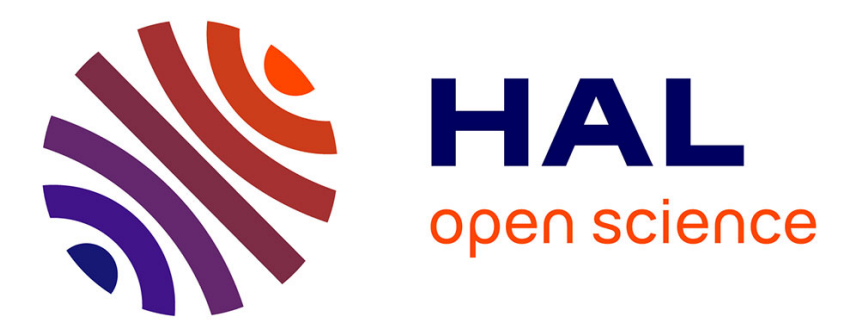

\title{
Application de la dilatométrie et des mesures magnétiques à l'étude de la transformation martensitique sous contrainte d'un alliage Fe-20 Ni-0,5 C

\author{
E. Gautier, A. Simon, G. Beck, G. Gavoille
}

\section{- To cite this version:}

E. Gautier, A. Simon, G. Beck, G. Gavoille. Application de la dilatométrie et des mesures magnétiques à l'étude de la transformation martensitique sous contrainte d'un alliage Fe-20 Ni-0,5 C. Revue de Physique Appliquée, 1987, 22 (11), pp.1371-1379. 10.1051/rphysap:0198700220110137100 . jpa00245687

\section{HAL Id: jpa-00245687 https://hal.science/jpa-00245687}

Submitted on 1 Jan 1987

HAL is a multi-disciplinary open access archive for the deposit and dissemination of scientific research documents, whether they are published or not. The documents may come from teaching and research institutions in France or abroad, or from public or private research centers.
L'archive ouverte pluridisciplinaire HAL, est destinée au dépôt et à la diffusion de documents scientifiques de niveau recherche, publiés ou non, émanant des établissements d'enseignement et de recherche français ou étrangers, des laboratoires publics ou privés. 


\title{
Application de la dilatométrie et des mesures magnétiques à l'étude de la transformation martensitique sous contrainte d'un alliage Fe-20 Ni-0,5 C
}

\author{
E. Gautier, A. Simon, G. Beck et G. Gavoille (*) \\ Laboratoire de Science et Génie des Matériaux Métalliques, Ecole des Mines, Parc de Saurupt, 54042 Nancy- \\ Cedex, France
}

(Reçu le 2 février 1987, accepté le 29 juillet 1987)

\begin{abstract}
Résumé. - Nous avons mis au point une méthode de suivi des variations de propriétés magnétiques pour l'étude de la transformation martensitique. Après étalonnage cette méthode nous permet d'atteindre directement la quantité de phase martensitique ferromagnétique formée. Nous avons couplé cette méthode à la dilatométrie pour étudier la transformation martensitique sous contrainte pour divers types de sollicitations mécaniques. Nous donnons des exemples quantitatifs des variations de la cinétique de transformation et de la plasticité de transformation pour la transformation martensitique d'un alliage $\mathrm{Fe}-20 \mathrm{Ni}-0,5 \mathrm{C}$ au cours d'essais de fluage anisotherme et d'essais de traction.
\end{abstract}

\begin{abstract}
We have devised a method for measuring the variations in magnetic properties in the study of martensitic transformation. After calibration we can directly measure the quantity of ferromagnetic martensite formed. This method has been combined with dilatometry in order to study martensitic transformation under stress for different kinds of mechanical tests. We give quantitative examples of the variations of transformation kinetics and measurements of deformation due to transformation plasticity for martensitic transformation of an Fe-20 Ni-0.5 C alloy during anisothermal creep tests and tensile tests.
\end{abstract}

\section{Introduction.}

Les mesures dilatométriques couramment utilisées pour l'étude des cinétiques de transformation de phase ne peuvent plus être corrélées à la quantité de phase formée lorsque la transformation a lieu sous contrainte. En effet, dans ces conditions il existe une plasticité de transformation dont la cause est liée à différents mécanismes [1-4] et dont les variations avec le taux de phase formée sont à l'heure actuelle peu connues.

L'étude des transformations sous contrainte, notamment la plasticité de transformation et l'effet des contraintes sur la cinétique de la transformation, nécessite en plus de la dilatométrie d'une seconde méthode d'étude plus caractéristique du changement de phase. Dans une première étape, nous avons utilisé les variations de résistivité [5-7]. Mais l'utilisation d'une telle méthode est difficile dans le cas de la transformation martensitique surtout lorsqu'il y a déformation de la phase mère [7].

(*) Laboratoire d'Electricité et d'Automatique, Université de Nancy I, B.P. 239, 54506 Nancy-Cedex, France.
Pour étudier les transformations martensitiques qui se produisent en dessous de la température ambiante, nous avons mis au point une nouvelle méthode d'étude liée aux variations des propriétés magnétiques de l'alliage. Nous présenterons des résultats obtenus pour un alliage $\mathrm{Fe}-20 \mathrm{Ni}-0,5 \mathrm{C}$ tant pour la cinétique de transformation que pour la plasticité de transformation.

\section{Méthode de suivi des variations de propriétés magnétiques.}

2.1 PRINCIPE DE LA MÉTHODE. - L'austénite des alliages $\mathrm{Fe}-\mathrm{Ni}-\mathrm{C}$ que nous étudions est paramagnétique et le produit transformé (martensite) est ferromagnétique.

Cette propriété a été utilisée par de nombreux auteurs pour détecter et suivre la transformation de phase $\gamma \rightarrow \alpha^{\prime}$ [8-10]. Lecroisey [9] et Abrassart [10] ont utilisé un appareillage constitué de deux bobines. La première induit un champ magnétique de 1800 OE. La seconde permet la mesure des variations de flux de l'échantillon en fonction de la déformation au cours des essais de traction. 
L'induction du matériau, calculée à partir de la variation du flux, est proportionnelle au taux de phase ferromagnétique formée lorsque le champ inducteur permet une mesure de l'induction à saturation.

Cette méthode présente un désavantage quant à la taille du dispositif expérimental. Elle nécessite des bobines donnant des champs élevés pour atteindre l'aimantation à saturation du matériau. Cependant, la propriété mesurée est directement proportionnelle au taux de phase martensitique formée.

La méthode utilisée par Olson et al. [8] est plus attrayante car la dimension du dispositif expérimental peut s'intégrer sur notre appareillage dont la description est présentée en références [11] et [6].

Avec quelques modifications, nous avons pu la réaliser en rendant le système très autonome et très compact.

Son principe est le suivant. L'échantillon constitue le noyau d'une bobine inductive. Cette bobine est insérée dans un circuit auto-oscillant.

Si l'inductance de la bobine varie, la fréquence de résonance du circuit varie. Aussi en suivant la fréquence de résonance, nous pouvons suivre les variations d'inductance de la bobine liées essentiellement aux variations de propriété magnétique de son noyau.

2.2 DesCRIPTION. - Le dispositif de mesure comporte deux parties; une bobine qui se place autour de l'échantillon et une partie électronique (alimentation de la bobine, traitement du signal).

Le corps de la bobine est réalisé en téflon. Le bobinage effectué avec du fil de cuivre de $0,3 \mathrm{~mm}$ de diamètre est constitué de 300 spires environ sur une longueur utile de $20 \mathrm{~mm}$. Cette longueur correspond à la partie utile de l'éprouvette.

La massiveté de la bobine limite l'efficacité du dispositif du refroidissement de l'échantillon. Aussi la bobine a-t-elle été réalisée afin d'assurer ellemême le refroidissement de l'échantillon.

\subsubsection{Alimentation de la bobine et traitement du} signal. - Nous avons réalisé un circuit oscillant sur la base du schéma décrit par Olson et al. [8]. La fréquence de résonance obtenue est convertie en tension à l'aide d'un circuit intégré convertisseur fréquence-tension 2917 N8.

Cette tension est ramenée à une valeur nulle pour une bobine dont le noyau est constitué d'un échantillon paramagnétique, puis elle est amplifiée.

Le schéma de cet ensemble est porté figure 1 où nous avons séparé les différents étages : circuit autooscillant, conversion fréquence-tension, amplification du signal.

Pour un échantillon ferromagnétique (totalement transformé) l'amplitude de variation du signal est de $8800 \mathrm{mV}$, ce qui conduit à une très bonne sensibilité

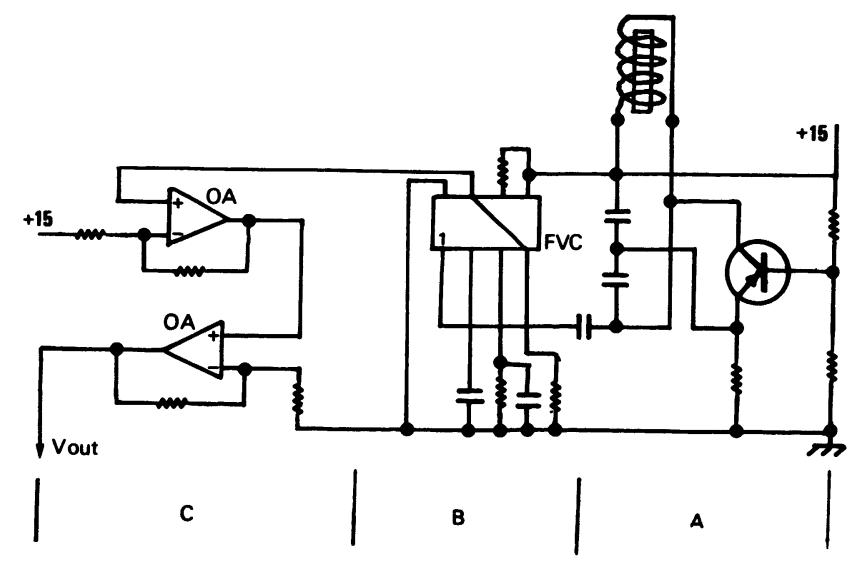

Fig. 1. - Schéma du dispositif de suivi des variations de propriétés magnétiques. A : Alimentation de la bobine ; $B$ : Convertion de la fréquence de résonance en tension continue ; C : Amplification du signal de sortie.

[Schematic diagram of the A.-C. magnetic technique used. A : Reel supplying; B : Conversion of resonance frequency into direct current ; C : Amplification of output signal.]

de la mesure vis-à-vis de la transformation martensitique.

2.2.2 Etalonnage. - Cette méthode ne permet pas de relier directement le paramètre mesuré à la quantité de phase ferromagnétique formée comme la méthode de mesure d'aimantation à saturation $[9,10]$. Aussi un étalonnage préalable à toutes mesures quantitatives est-il nécessaire.

Le dispositif a été étalonné in situ sur notre appareillage : c'est-à-dire différents échantillons ont été refroidis à différentes températures puis rapidement réchauffés à température ambiante. La quantité de phase transformée est alors mesurée par aimantation à saturation. La figure 2 montre la courbe d'étalonnage obtenue, taux de variation du signal de sortie de notre dispositif $(\Delta V)$ (par rapport à la valeur maximale de 8,8 volts) en fonction du taux de phase martensitique mesuré par aimantation à saturation. Nous observons une corrélation linéaire entre ces deux paramètres ; c'est-à-dire que notre méthode de mesure donne un signal dont les variations sont proportionnelles à la quantité de martensite formée. De légères variations sont observées aux faibles taux de phase transformée mais ceci peut être lié à une inhomogénéité de la structure le long de l'éprouvette due à de faibles écarts de température $\left(<5^{\circ} \mathrm{C}\right.$ sur la partie utile dont la longueur est $20 \mathrm{~mm}$ ). Les mesures d'aimantation à saturation s'effectuent sur la partie centrale $(\simeq 3 \mathrm{~mm}$ de longueur).

Nous avons de plus mesuré le taux de phase formée par analyse d'image quantitative. Cette méthode est moins précise que la méthode de 


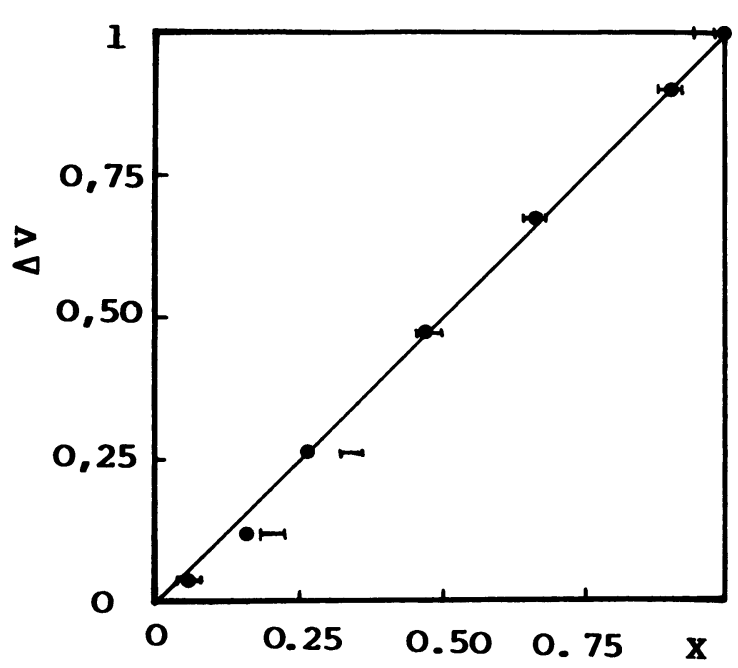

Fig. 2. - Courbe d'étalonnage pour un alliage Fe-Ni-C. Signal de sortie $\Delta V$ normalisé à l'unité, en fonction du taux de phase formée $X$. $X$ est mesuré : • Par aimantation à saturation $; \longmapsto$ Par analyse d'image.

[Output signal normalized to unity $(\Delta V)$ versus the content of phase formed $X . X$ is measured $\bullet$ by bulk magnetization ; by quantitative image analysing.]

mesure du taux de martensite par aimantation à saturation utilisée préalablement. Les résultats obtenus confortent dans l'ensemble l'étalonnage précédent.

Nous pouvons donc directement lier le taux de variation du signal au taux de martensite formée : $\Delta V=X$.

Au cours de nos essais, deux sources de variation du signal peuvent exister en plus de la transformation de phase. Pour des essais à température variable, une variation de résistance électrique de la bobine avec la température conduit à une variation du signal de $1,35 \mathrm{mV} /{ }^{\circ} \mathrm{C}$. Cette valeur est faible et elle est équivalente au signal produit par la formation de $0,015 \%$ de martensite. Dans le dépouillement des essais nous avons cependant tenu compte de cette variation du signal de la bobine avec la température.

Cette méthode présente aussi une sensibilité à la déformation de l'éprouvette. Nous avons mesuré la variation du signal obtenu au cours de la traction de différents matériaux (paramagnétique et ferromagnétique). Les variations de signal sont présentées figure 3 où nous avons porté la variation du signal comme un équivalent de taux de transformation en fonction de la déformation de l'échantillon.

Dans le cas de l'échantillon paramagnétique (acier inoxydable à $18 \% \mathrm{Cr}$ et $8 \% \mathrm{Ni}$, essai à $20^{\circ} \mathrm{C}$ ) la variation observée est faible $\Delta X / \Delta \varepsilon=0,035$ et n'est observée que dans le domaine de déformation plastique (courbe a). Une part de cet effet doit être attribuée à un début de transformation provoquée par la déformation elle-même. L'échantillon ferromagnétique testé est l'alliage $\mathrm{Fe}-\mathrm{Ni}-\mathrm{C}$, trempé à

\footnotetext{
REVUE DE PHYSIQUE APPLIQUÉE. - T. 22, N 11, NOVEMBRE 1987
}

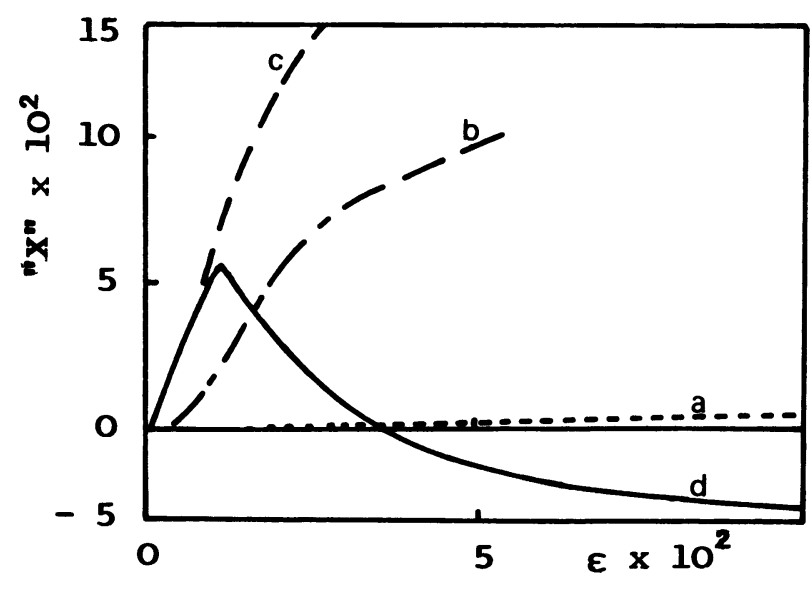

Fig. 3. - Sensibilité de la méthode à la déformation. Signal de sortie porté comme un taux équivalent de phase transformée, en fonction de la déformation. a) Acier austénitique 18-8 (paramagnétique). b) Alliage Fe-Ni-C partiellement transformé. c) Alliage $\mathrm{Fe}-\mathrm{Ni}-\mathrm{C}$ martensitique (ferromagnétique). d) Acier à $0,8 \%$ de carbone (ferromagnétique).

[Sensibility of the method to the deformation. Output signal variations as an equivalent of content of phase formed versus deformation. a) Austenitic stainless steel 18-8 (paramagnetic). b) Fe-Ni-C alloy partly transformed. c) $\mathrm{Fe}-\mathrm{Ni}-\mathrm{C}$ alloy transformed in martensite (Ferromagnetic). d) Eutectoid steel (Ferromagnetic).]

l'hélium liquide dont la structure est quasiment martensitique (courbe b). Il n'a été sollicité que dans le domaine de déformation élastique à $20^{\circ} \mathrm{C}$. La variation $\Delta X / \Delta \varepsilon$ est toujours positive $(\Delta X / \Delta \varepsilon \simeq 5)$. Un alliage partiellement transformé (courbe c) présente dans le domaine de déformation élastique une pente positive plus faible que l'alliage quasiment martensitique. Dans le domaine de déformation plastique, de la martensite se forme au cours de l'essai nous ne pouvons plus définir le sens de variation du signal avec uniquement la déformation plastique.

Pour connaître le sens de variation de notre signal avec la déformation pour un échantillon ferromagnétique, nous avons testé un acier eutectoïde (courbe d). Dans le domaine de déformation élastique, nous obtenons les mêmes variations que pour l'alliage FeNi-C quasiment martensitique. Par contre, dans le domaine de déformation plastique, le sens de variation s'inverse. Les valeurs $\Delta X / \Delta \varepsilon$ varient de $-\dot{2,27}$ à $-0,45$ lorsque la déformation augmente.

Nous voyons que le matériau paramagnétique ne présente pas ou très peu de sensibilité à la contrainte/déformation. Par contre le matériau ferromagnétique est sensible à la contrainte ou à la déformation. L'origine de ces sensibilités est discutée en [12] ; nos résultats sont proches de ceux observés pour le fer ou le permalloy [12].

Pour nos essais sur la transformation martensiti- 
que, une correction liée à cet effet est difficile à introduire car le taux de phase varie et la sensibilité à la déformation varie continuement entre les valeurs définies par l'échantillon paramagnétique et celles définies pour l'alliage ferromagnétique qui se déforme plastiquement. Aussi nous ne ferons pas de correction liée à la déformation plastique. Cependant l'erreur maximale introduite est estimée à la fin de l'essai en comparant la valeur donnée par cette méthode et celle que nous avons mesurée par analyse d'image quantitative. Les écarts sont faibles dans notre cas, pour l'essai présenté par la suite le taux de martensite mesuré par méthode magnétique est de $65 \%$. Celui mesuré par analyse d'image est de 66 à $70 \%$.

Certaines mesures ne seront pas du tout ou très peu entachées d'erreurs, notamment la détermination de paramètres critiques au début de la transformation et les premiers pourcentages de phase martensitique.

En conclusion, nous voyons que cette méthode nous permet de suivre les transformations de phase au cours de refroidissement sous contrainte ou non, ou au cours d'essai de traction.

Nous obtenons un suivi continu de la transformation où le taux de variation du signal est proportionnel au taux de phase ferromagnétique formée.

\section{Application.}

Cette méthode est utilisée pour l'étude des transformations martensitiques sous contraintes de traction, contraintes qui vont modifier la cinétique de transformation et produire une déformation plastique liée à cette transformation. Nous donnons ici en exemple une étude quantitative sur la cinétique de transformation et sur la plasticité de transformation qui a ainsi pu être réalisée en combinant mesures dilatométriques et mesures de propriétés magnétiques.

Des essais de fluage anisotherme et de traction ont été effectués pour l'alliage dont la composition est donnée dans le tableau I.

3.1 Mesures effectuées. - Les figures 4, 5, 6 montrent respectivement les résultats expérimentaux obtenus au cours d'un essai de fluage anisotherme (charge constante et température variable) et au cours d'essais de traction à vitesse de déformation constante et à vitesse de mise en charge constante.

Pour les essais de fluage anisotherme (Fig. 4),

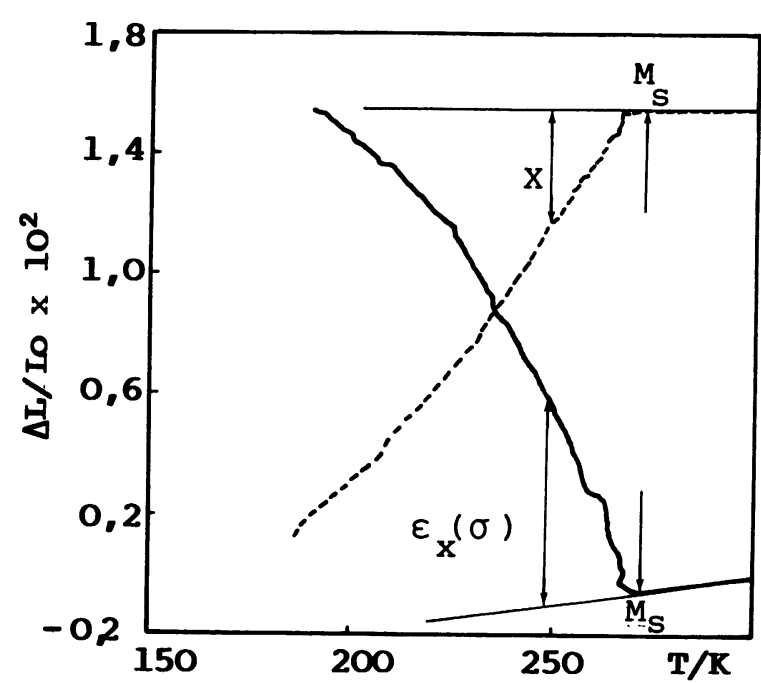

Fig. 4. - Courbes expérimentales au cours d'un essai de fluage anisotherme. Variations du taux de phase formée $X$ et de l'allongement $\Delta L / L_{0}$ en fonction de la température $T$.

[Experimental recordings during an anisothermal creep test. Variations of content of phase formed $X$ and elongation $\Delta L / L_{0}$ versus temperature $T$.]

nous avons enregistré les variations de propriétés magnétiques et les variations d'allongement en fonction de la température. Sur ces courbes, nous observons la tempétature $M_{\mathrm{s}}$ sous contrainte par les deux méthodes. Nous obtenons directement la cinétique de transformation en fonction de la température à partir des courbes variation de propriétés magnétiques en fonction de la température.

De plus, à chaque taux de phase formée $X$, nous pouvons associer un taux de déformation $\varepsilon_{X}(\sigma)$, pour la contrainte appliquée $\sigma$.

Nous pouvons alors atteindre la déformation de plasticité de transformation qui est égale à $\left(\varepsilon_{X}(\sigma)-\varepsilon_{X}(0)\right)$ pour le taux de phase formée $X$.

$\varepsilon_{X}(0)$ est la déformation associée au taux de phase formée $X$ pour une transformation sans contrainte appliquée.

Pour l'essai de fluage anisotherme, la combinaison dilatométrie - propriétés magnétiques nous conduit aux données suivantes :

- température de début de transformation $M_{\mathrm{s}}$,

- cinétique de transformation sous charge constante en fonction de la température $X=f(T)$ à $\sigma$ donnée,

Tableau I. - Composition de l'alliage Fe-20 Ni-0,5 C (\% pondéral) et température $M_{\mathrm{s}}$. [Composition (in wt \%) and $M_{\mathrm{S}}$ temperature of the $\mathrm{Fe}-20 \mathrm{Ni}-0.5 \mathrm{C}$ alloy.]

\begin{tabular}{|c|c|c|c|c|c|c|c|c|c|c|}
\hline $\mathrm{C}$ & $\mathrm{Ni}$ & $\mathrm{Cr}$ & $\mathrm{Mo}$ & $\mathrm{Mn}$ & $\mathrm{Si}$ & $\mathrm{P}$ & $\mathrm{S}$ & $\mathrm{Fe}$ & $M_{\mathrm{s}}$ & Austénisation \\
\hline 0,48 & 19,29 & 0,02 & 0,01 & 0,25 & 0,16 & 0,015 & 0,021 & bal & $-6{ }^{\circ} \mathrm{C}$ & $\begin{array}{c}20 \text { min à } \\
1000^{\circ} \mathrm{C}\end{array}$ \\
\hline
\end{tabular}



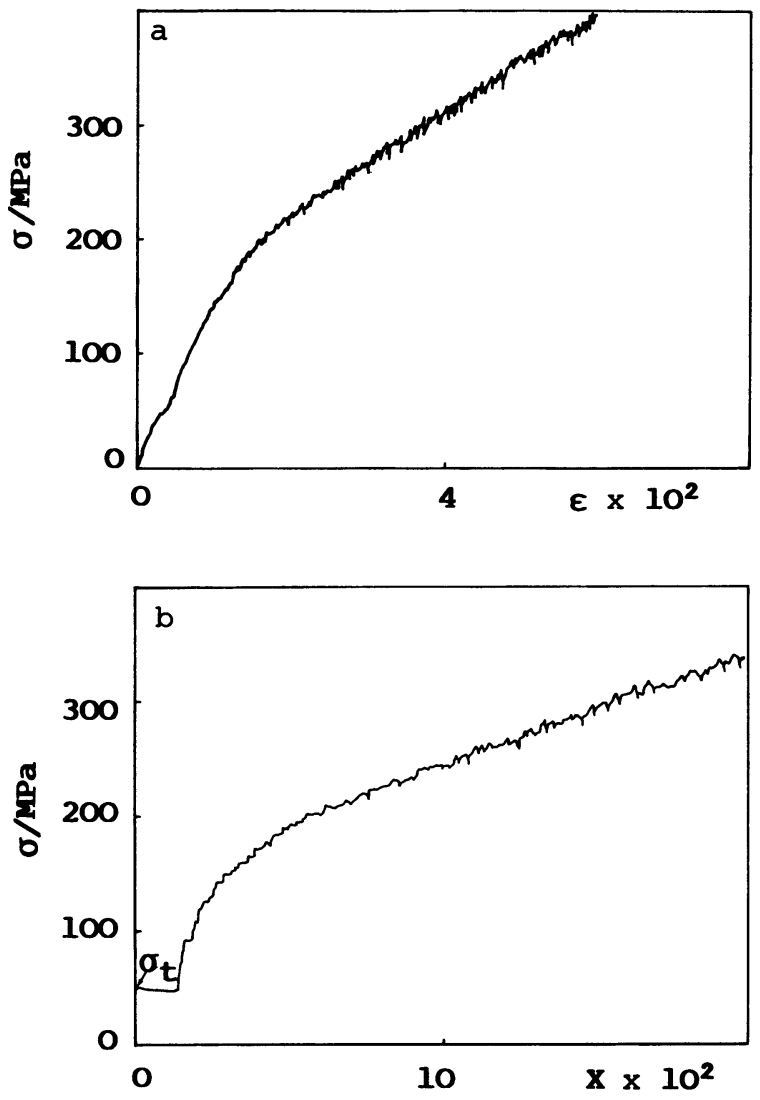

Fig. 5. - Enregistrements expérimentaux au cours d'un essai de traction à vitesse de déformation constante. La contrainte appliquée $\sigma$ est enregistrée en fonction de la déformation de l'échantillon $\varepsilon$ (courbe a) et du taux de phase transformée $(X)$ (courbe $b$ ).

[Experimental recordings during a tensile test at constant deformation rate. The applied stress $\sigma$ is recorded versus the sample deformation $\varepsilon$ (curve a) and the content of phase formed $X$ (curve b).]

- déformation de plasticité de transformation en fonction de l'avance de la réaction pour une contrainte $\sigma\left(\varepsilon_{X}(\sigma)-\varepsilon_{X}(0)\right)=f(X)$.

Dans le cas des essais de traction, nous obtenons simultanément les courbes de traction contrainte en fonction de la déformation $\sigma=f(\varepsilon)$ (Figs. 5a et 6a) et les courbes contrainte en fonction du taux de transformation $\sigma=f(\Delta V=X)$ (Figs. 5b et 6b).

Ces essais de traction sont réalisés à une température supérieure à la température $M_{s}$. Dans ce cas, la transformation peut être assistée/induite par la contrainte/déformation [13]. Il existe une contrainte critique $\sigma_{t}$ pour laquelle la transformation aura lieu. Cette contrainte peut être définie sans équivoque sur les courbes $\sigma=f(X)$ alors que sur les courbes $\sigma=f(\varepsilon)$ sa détermination est plus délicate.

L'analyse des courbes $\sigma=f(\varepsilon)$ et $\sigma=f(X)$ montre un comportement différent lorsque l'alliage est sollicité à vitesse de déformation constante ou à vitesse de mise en charge constante.
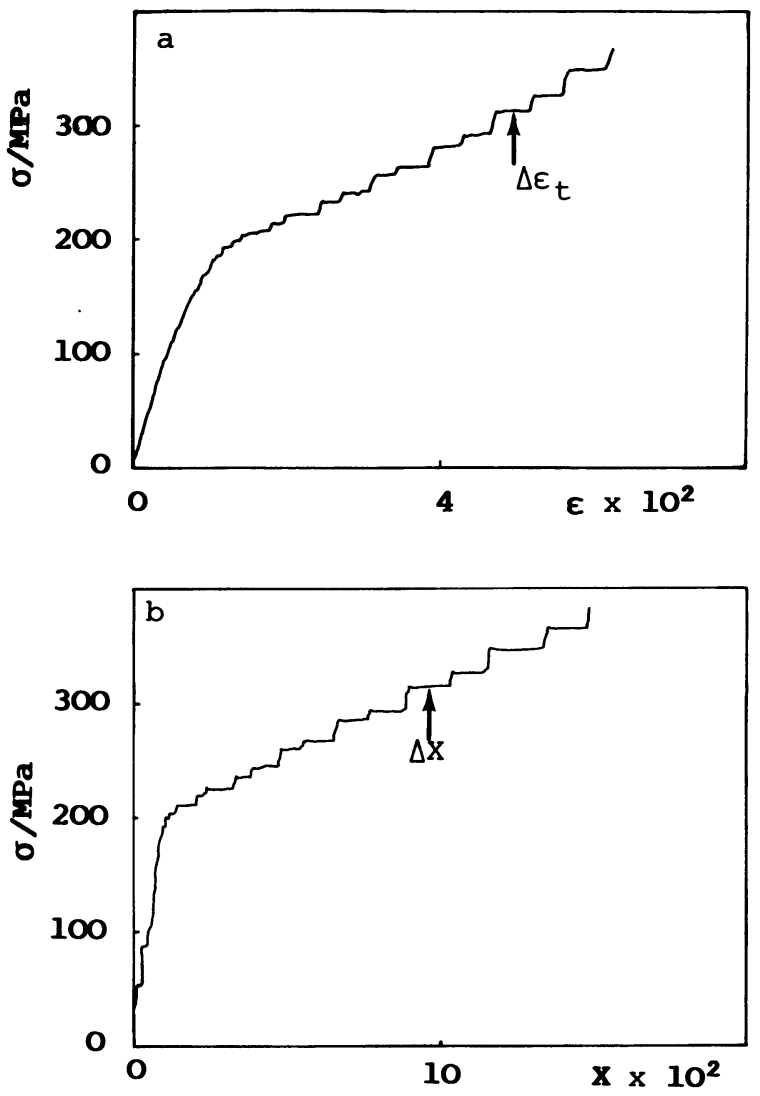

Fig. 6. - Enregistrements expérimentaux au cours d'un essai de traction à vitesse de mise en charge constante. La contrainte appliquée $\sigma$ est enregistrée en fonction de la déformation de l'échantillon $\varepsilon$ (courbe a) et du taux de phase transformée $X$ (courbe b).

[Experimental recordings during a tensile test at constant loading rate. The applied stress $\sigma$ is recorded versus the sample deformation $\varepsilon$ (curve a) and the content of phase formed $X$ (curve b).]

A vitesse de déformation constante, la transformation se traduit par une relaxation des contraintes, à vitesse de mise en charge constante elle se traduit par une déformation très rapide à charge constante. Pour ces essais, nous pouvons suivre la cinétique de transformation en fonction de la contrainte ou de la déformation. Par contre, il est plus difficile de déterminer la déformation de plasticité de transformation pour les essais à vitesse de déformation constante. Dans certains cas, il est possible d'assimiler la déformation de transformation à la déformation nécessaire pour atteindre le niveau de contrainte avant relaxation lorsque celle-ci a eu lieu, comme l'ont fait certains auteurs [14]. Dans notre cas, le dépouillement complet de la courbe par cette méthode est très imprécis.

Par contre, pour les essais à vitesse de mise en charge constante, l'essentiel de la transformation se fait au cours des « paliers de charge ». A l'incrément de transformarion $\Delta X$, nous pouvons associer une déformation $\Delta \varepsilon_{\mathrm{t}}$ que nous attribuons seulement à la 
transformation. (La charge étant constante, il n'y a pas de déformation plastique au sens classique du terme.)

La déformation de plasticité de transformation peut alors être définie comme étant la valeur :

$$
\Delta \varepsilon_{\mathrm{t}}-\varepsilon_{0} \Delta X / 3
$$

où $\varepsilon_{0}$ est la variation volumique liée à la transformation de phase.

La mesure de la déformation de plasticité de transformation totale peut être obtenue en fonction de $X$ en cumulant les valeurs $\left(\Delta \varepsilon_{t}-\varepsilon_{0} \Delta X / 3\right)$ et en les portant en fonction de $X$.

En résumé, les données obtenues à partir de ces courbes sont :

- la contrainte critique $\sigma_{\mathrm{t}}$ pour assister/induire la transformation ;

- la cinétique de transformation en fonction de la contrainte ou de la déformation;

- la déformation de plasticité de transformation en fonction de l'avancement de la réaction.

3.2 EXPLOITATION DES ESSAIS. - La mesure magnétique nous a permis de déterminer l'influence des contraintes sur la cinétique de transformation variation de température $M_{\mathrm{s}}$, variation de l'avancement de la transformation avec la contrainte. Connaissant la quantité de martensite formée en cours d'essai, nous avons pu développer des études quantitatives sur la plasticité de transformation.

Nous allons présenter dans une première partie la variation de la température de début de transformation $M_{\mathrm{s}}$ avec la contrainte appliquée pour des essais de fluage anisotherme ou de traction et dans une seconde partie les mesures de déformation de plasticité de transformation pour ces mêmes types d'essais.

\subsubsection{Influence de la contrainte sur la température de} début de transformation $M_{\mathrm{s}}$. - En essais de fluage anisotherme, la contrainte est appliquée à l'échantillon à une température supérieure à $M_{\mathrm{s}}$ et la transformation se produit seulement au cours du refroidissement. Nous avons porté figure 7 les variations de la température $M_{\mathrm{s}}$ avec la contrainte appliquée. Nous observons pour des contraintes inférieures à $160 \mathrm{MPa}$ une augmentation linéaire de la température $M_{\mathrm{s}}$ avec la contrainte appliquée. Pour des contraintes supérieures à cette valeur, les variations de $M_{\mathrm{s}}$ avec la contrainte s'inversent. Puis pour des contraintes plus élevées $\mathrm{d} M_{\mathrm{s}} / \mathrm{d} \sigma$ est à nouveau positif.

Lorsque la contrainte est inférieure à $160 \mathrm{MPa}$, les résultats obtenus sont proches de ceux prévus par les modèles de Patel et Cohen [15] ou Wollants [16]. La pente $\mathrm{d} M_{\mathrm{s}} / \mathrm{d} \sigma$ est égale à $0,13^{\circ} \mathrm{C} / \mathrm{MPa}$. (Les modèles prévoient une valeur de $0,14^{\circ} \mathrm{C} / \mathrm{MPa}$ [17].)

Pour les essais à contrainte supérieure à $160 \mathrm{MPa}$, une légère déformation plastique s'est produite lors

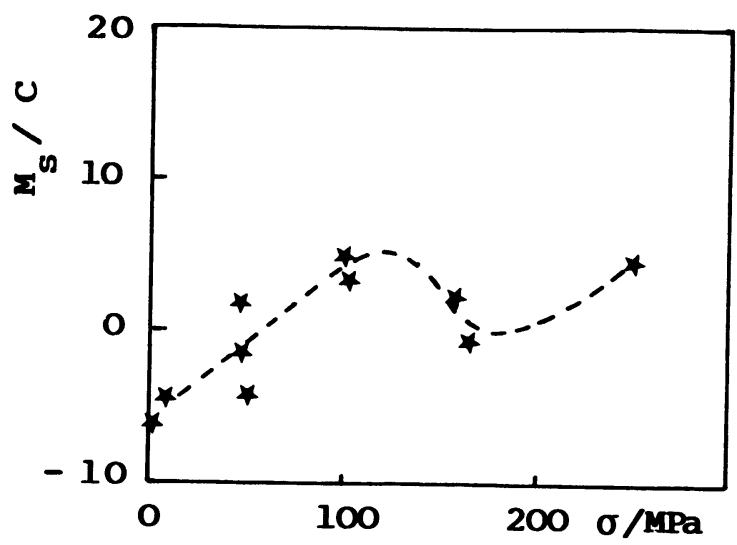

Fig. 7. - Variations de $M_{\mathrm{S}}$ avec la contrainte appliquée au cours d'essais de fluage anisotherme.

$\left[M_{\mathrm{S}}\right.$ variations versus applied stress during anisothermal creep tests.]

de la mise sous contrainte à température supérieure à $M_{\mathrm{s}}$. La consolidation introduite par cette déformation plastique peut expliquer l'inversion dans la pente $\mathrm{d} M_{\mathrm{s}} / \mathrm{d} \sigma$. La phase mère étant consolidée, l'énergie de déformation associée à la transformation va augmenter. Il en résulte une diminution de la température $M_{\mathrm{s}}[18,19]$ par rapport à la valeur attendue si l'on considère l'effet de la contrainte seule qui est d'augmenter la température $M_{\mathrm{s}}$. L'effet d'une déformation plastique préalable a été étudiée et différents auteurs [19-22] ont observé une diminution de la température $M_{\mathrm{s}}$ pour de faibles déformations ( 3 à $5 \%$ ) et une augmentation de $M_{\mathrm{s}}$ pour des déformations plus importantes.

Pour l'essai à $250 \mathrm{MPa}$, la température $M_{\mathrm{s}}$ est telle que $\mathrm{d} M_{\mathrm{s}} / \mathrm{d} \sigma$ est à nouveau positif. Dans ce cas, la déformation étant plus importante, elle joue un second rôle, celui d'aide à la transformation.

Dans le cas des essais de traction, nous avons porté figure 8 , la contrainte critique $\sigma_{\mathrm{t}}$ qui provoque la transformation en fonction de la température de l'essai $T$. Pour les températures supérieures ou égales à $0{ }^{\circ} \mathrm{C}$, nous observons une relation sensiblement linéaire entre $\sigma_{\mathrm{t}}$ et $T$ jusqu'à la température de $20^{\circ} \mathrm{C}$ environ. Cette contrainte critique est inférieure à la limite d'élasticité à $0,2 \%$. Au-delà de $20^{\circ} \mathrm{C}$, la contrainte critique est supérieure à la limite d'élasticité, c'est-à-dire que le matériau est plastiquement déformé avant de se transformer.

Ce résultat entre dans le schéma bien établi de transformation martensitique assistée par la contrainte ou induite par la déformation [13]. Dans le domaine de variation linéaire de $\sigma_{t}$ avec la température, la transformation martensitique est assistée par la contrainte, nous observons des variations $\mathrm{d} \sigma_{\mathrm{t}} / \mathrm{d} T$ équivalentes aux variations de $M_{\mathrm{s}}$ observées en fluage anisotherme aux contraintes à $160 \mathrm{MPa}\left(\mathrm{d} T / \mathrm{d} \sigma_{\mathrm{t}}=0,13^{\circ} \mathrm{C} / \mathrm{MPa}\right)$. Pour ce domaine de température, le seul rôle de la contrainte est 


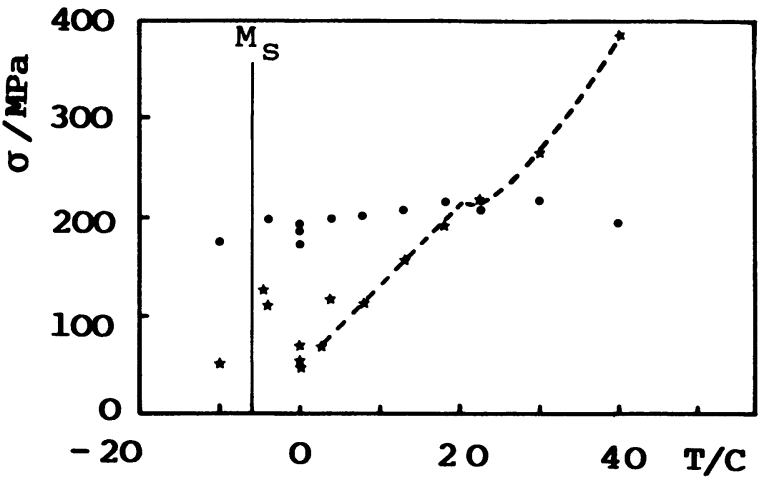

Fig. 8. - Variations de la contrainte critique $\sigma_{t}(\star)$ pour induire la transformation en fonction de la température de l'essai (essais de traction). (•) Valeurs de la contrainte lorsque $\varepsilon=0,002$.

[Variations of critical stress $\sigma_{\mathrm{t}}(\star)$ to induce transformation versus test temperature (tensile tests). (•) Stress values for $\varepsilon=0.002$.]

d'apporter une force motrice supplémentaire à la transformation.

Pour les températures supérieures à $20^{\circ} \mathrm{C}$, domaine de transformation martensitique aidée par la déformation, nous retrouvons le deuxième rôle invoqué précédemment pour les essais de fluage anisotherme, rôle d'aide de la déformation à la transformation. Deux interprétations ont été proposées confortées toutes deux par des résultats expérimentaux ou des observations microscopiques [13, 23-27].

i) la déformation crée des contraintes internes qui vont localement atteindre la valeur de la contrainte critique et provoquer la transformation [23-26] ;

ii) des sites préférentiels de germination sont créés par la déformation : intersection de bandes de cisaillement, de déformation [27, 13]. Ces deux origines peuvent exister et être intimement liées surtout lorsque la déformation plastique s'est produite avant la transformation dans un domaine ou l'alliage ne se transforme pas.

Si nous comparons les deux types d'essais, dans le cas de l'essai de traction les faibles déformations ont un rôle d'aide à la transformation. Par contre en essai de fluage anisotherme elles inhibent la transformation. Le mode de sollicitation joue un grand rôle. Ce phénomène est discuté par ailleurs $[17,28]$ nous l'attribuons à une plus grande facilité d'accommodation des déformations de transformation par déformation plastique de l'austénite.

\subsubsection{Plasticité de transformation.}

Plasticité de transformation en fluage anisotherme. - Les courbes de déformation de plasticité de transformation en fonction du taux d'avancement de la réaction sont présentées figure 9 pour des contraintes variant de 50 à $250 \mathrm{MPa}$. Nous observons

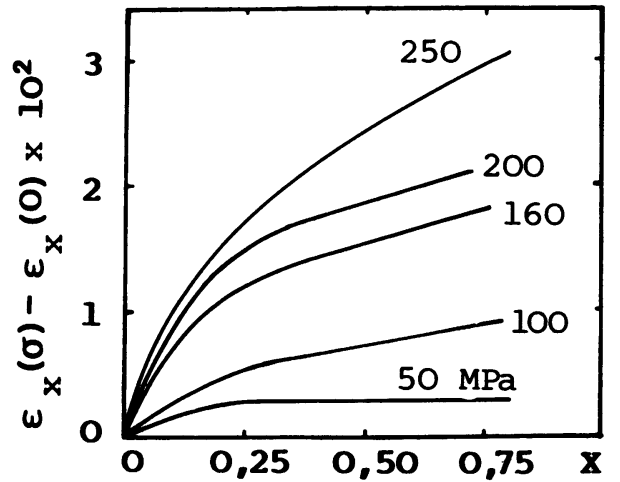

Fig. 9. - Déformation de plasticité de transformation en fonction du taux de phase formée pour différentes contraintes appliquées. Essais de fluage anisotherme.

[Deformation due to transformation plasticity versus content of phase formed for different constant applied stresses. Anisothermal creep tests.]

une augmentation rapide de la déformation de plasticité de transformation au début de la réaction puis, la déformation de plasticité de transformation varie linéairement avec le taux de phase transformée.

Nous avons porté dans le tableau II les valeurs des pentes initiales et des pentes des parties linéaires. Enfin, nous avons également porté cette dernière valeur divisée par la contrainte appliquée.

Les pentes initiales augmentent très rapidement pour atteindre des valeurs égales à 0,12 pour les plus fortes contraintes appliquées. Ces pentes sont proches pour les contraintes de 200 et $250 \mathrm{MPa}$. Pour la partie linéaire, les pentes $\frac{\mathrm{d} \varepsilon_{\mathrm{pt}}}{\mathrm{d} X}$ augmentent égale-

Tableau II. - Variations des pentes initiales et des pentes de la partie linéaire des courbes de plasticité de transformation fonction du taux de transformation, pour différentes contraintes appliquées.

[Variations of the slopes of the curves transformation plasticity deformation function of transformation content, in the initial stage and the linear stage, for different applied stresses.]

\begin{tabular}{|c|l|c|c|}
\hline $\begin{array}{c}\text { Contrainte } \\
\text { appliquée } \\
\mathrm{MPa}\end{array}$ & $\frac{\mathrm{d} \varepsilon_{\mathrm{pt}}}{\mathrm{d} X}$ initial & $\frac{\mathrm{d} \varepsilon_{\mathrm{pt}}}{\mathrm{d} X}$ linéaire & $\begin{array}{c}\frac{\mathrm{d} \varepsilon_{\mathrm{pt}}}{\mathrm{d} X} \cdot \frac{1}{\sigma} \\
\mathrm{MPa}^{-1} \text { lin }\end{array}$ \\
\hline 50 & 0,016 & 0 & 0 \\
\hline 100 & 0,04 & $0,65 \times 10^{-2}$ & $6,5 \times 10^{-5}$ \\
\hline 160 & 0,09 & $1,08 \times 10^{-2}$ & $6,75 \times 10^{-5}$ \\
\hline 200 & 0,104 & $1,14 \times 10^{-2}$ & $5,6 \times 10^{-5}$ \\
\hline 250 & 0,12 & & \\
\hline
\end{tabular}


ment avec la contrainte appliquée. Si ces valeurs sont divisées par la contrainte appliquée, le rapport $\left(\frac{\mathrm{d} \varepsilon_{\mathrm{pt}}}{\mathrm{d} X}\right)\left(\frac{1}{\sigma}\right)$ est sensiblement constant, et vaut environ $6 \times 10^{-5} \mathrm{MPa}^{-1}$.

L'origine de la plasticité de transformation est attribuée à deux mécanismes.

- Orientation des plaquettes de martensite. Mécanisme de Magee [4].

- Ecoulement plastique supplémentaire dans le sens de la contrainte appliquée dû à la superposition du champ de contraintes internes lié à la déformation de transformation $\left(\frac{\Delta V}{V}, \gamma\right)$, au champ de contrainte externe appliqué $[2,3,29]$. Mécanisme de Greenwood-Johnson.

La déformation maximale liée à l'orientation des plaquettes de martensite peut être calculée à partir de la relation suivante (4) :

$$
\varepsilon_{\mathrm{t}}=\frac{1}{2}\left[\gamma_{0} \sin 2 \theta \cos \alpha+\varepsilon_{0}(1+\cos 2 \theta)\right]
$$

où

$\gamma_{0}$ est la déformation de cisaillement.

$\varepsilon_{0}$ la variation volumique.

$\theta$ l'angle entre la normale au plan d'habitat de la plaquette de martensite et la direction de la contrainte appliquée.

$\alpha$ l'angle entre la direction du cisaillement et la direction de cisaillement maximale.

$\varepsilon_{\mathrm{t}}$ est maximale considérant $\alpha=0$, lorsque $\operatorname{tg} 2 \theta$ est égal à $\gamma_{0} / \varepsilon_{0}$.

Pour notre alliage, $\varepsilon_{0}=3 \varepsilon_{\mathrm{X}}(0)$ lorsque $X=1$ et en considérant la variation volumique isotrope. $\mathrm{La}$ valeur obtenue est 0,024 .

Le calcul de $\varepsilon_{\mathrm{t}}$ maximale conduit à une valeur de 0,11 à partir de la valeur $\varepsilon_{0}$ obtenue expérimentalement et de la valeur $\gamma_{0}=0,19$ donnée par Pradhan [30] pour un alliage de composition voisine.

Cette valeur de 0,11 est proche des pentes initiales obtenues pour des contraintes de 160 à $250 \mathrm{MPa}$. Ces valeurs de pentes initiales ainsi que les variations de plasticité de transformation avec la contrainte obtenues pour de faibles taux de transformations [31], nous conduisent à proposer que le mécanisme de Magee est important au début de la réaction. La contribution de ce mécanisme diminue lorsque la transformation progresse.

La contribution du mécanisme d'orientation des plaquettes diminuant, les parties linéaires des courbes peuvent être l'image du mécanisme de «Greenwood-Johnson ». La variation de la plasticité de transformation serait alors une fonction linéaire du taux de phase formée et de la contrainte appliquée $\left(\left(\frac{\mathrm{d} \varepsilon_{\mathrm{pt}}}{\mathrm{d} X}\right)\left(\frac{1}{\sigma}\right)\right.$ est sensiblement constant). Ce résultat est similaire à celui que nous avons obtenu pour une transformation perlitique où la plasticité de transformation varie linéairement avec le taux de phase formée et la contrainte appliquée [32]. Dans le cas de ce dernier type de transformation, seul le mécanisme de «Greenwood-Johnson » est opérant.

Plasticité de transformation en essai de traction. Nous avons porté figure 10 la courbe de déformation de plasticité de transformation en fonction du taux d'avancement de la réaction pour un essai de traction à $0^{\circ} \mathrm{C}$. La plasticité de transformation est obtenue à partir de l'essai à vitesse de mise en charge constante, ainsi que nous l'avons défini par sommation des incréments de déformation de plasticité de transformation.

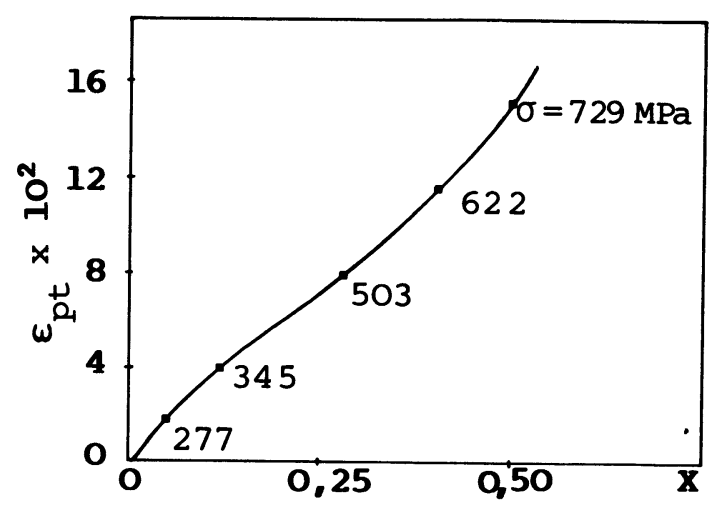

Fig. 10. - Déformation de plasticité de transformation en fonction du taux de phase formée durant un essai de traction à $0^{\circ} \mathrm{C}$.

[Deformation due to transformation plasticity versus the content of phase formed during a tensile test at $0{ }^{\circ} \mathrm{C}$.]

La contrainte augmente au cours de l'essai et nous avons porté certaines valeurs de contrainte sur la courbe de déformation de plasticité de transformation. Si nous comparons ce résultat à la déformation obtenue en fluage anisotherme, nous observons une plasticité beaucoup plus importante. Pour $X=0,45$, la déformation en essai de traction est de 0,13 alors qu'en essai de fluage anisotherme elle est de 0,024 pour ce même taux de transformation et une contrainte de $250 \mathrm{MPa}$. Comme la contrainte augmente tout au long de l'essai de traction, nous arrêtons notre comparaison à la déformation plastique obtenue lorsque la contrainte atteint $277 \mathrm{MPa}$. Nous avons encore un écart d'un facteur 3 entre la plasticité obtenue en essai de traction pour une contrainte variant de 0 à $277 \mathrm{MPa}$ et celle obtenue pour l'essai de fluage où la contrainte est constante et égale à $250 \mathrm{MPa}$. Les domaines de température sont également voisins.

La comparaison sommaire des résultats obtenus pour ces deux essais, fluage anisotherme-traction, montre des comportements en plasticité de transformation très différents pour des valeurs de contrainte et températures voisines qui ne peuvent être simple- 
ment expliquées par une contribution différente des deux mécanismes tels qu'ils apparaissent en essai de fluage anisotherme.

Une comparaison détaillée de ces comportements a été faite par ailleurs ainsi qu'une interprétation de cette différence $[17,28,31]$.

\section{Conclusion.}

La combinaison de la dilatométrie et des mesures de propriétés magnétiques nous a permis d'étudier quantitativement l'influence des contraintes sur la transformation martensitique. La méthode magnétique nous a permis de suivre avec précision le déroulement de la transformation martensitique (variation de la température $M_{\mathrm{s}}$ et progression de la transformation) pour différents types de sollicitations thermomécaniques de l'austénite. Associée à la dilatométrie, cette méthode nous permet de relier quantitativement la déformation de plasticité de transformation au taux de martensite formée et ceci pour divers essais thermomécaniques. En essai de fluage anisotherme la déformation de plasticité de transformation est importante au début de transformation et diminue progressivement. Elle varie linéairement avec la contrainte appliquée et l'avancement de la transformation à partir d'environ $25 \%$ de transformation. La plasticité mesurée en essai de traction est bien plus élevée qu'en essai de fluage alors que les conditions contrainte-température sont voisines. Ces mesures de la cinétique de transformation et de la déformation de plasticité de transformation rendues possibles grâce à l'emploi des deux méthodes d'études complémentaires permet d'aller au-delà de la simple mesure du phénomène, en situant la contribution respective des différents mécanismes responsables de la plasticité de transformation. Une discussion approfondie de ces mécanismes et une comparaison aux travaux existants est menée par ailleurs.

\section{Bibliographie}

[1] De Jong, M. and Rathenau, G. W., Acta Metall. 7 (1959) 246.

[2] De Jong, M. and Rathenau, G. W., Acta Metall. 9 (1961) 714.

[3] Greenwood, G. W. and Johnson, R. H., Proc. $R$. Soc. 283A (1965) 403.

[4] Magee, C. L., Ph. D. Thesis Carnegie Mellon University 1966.

[5] Simon, A., Gautier, E., Collette, G. and Beck, G., Bulletin du Cercle d'Etudes des Métaux, Tome XIV (1979) VI-1.

[6] Gautier, E., Hazotte, A., Simon, A. and Beck, G., $24^{\mathrm{e}}$ Colloque de Métallurgie «Les traitements Thermomécaniques » I.N.S.T.N. Saclay (1981) 187.

[7] Simon, A., Collette, G., Gautier, E. and Beck, G., Res. Mec. Lett. 1 (1981) 207.

[8] Olson, G. B., Azrin, M. and Band, H. E., A.M.M.R.C. Report TR 77-10 (1977).

[9] LeCroisey, F., Thèse de Doctorat ès-Sciences Physiques, Université de Nancy I, 1971.

[10] Abrassart, F., Thèse de Doctorat ès-Sciences Physiques, Université de Nancy I, 1972.

[11] Simon, A., BouXirot, F., GAutier, E. and BeCK, G., Mém. Sci. Rev. Metall. 75 (1978) 33.

[12] Bozorth, R. M., Ferromagnetism (D. Van Nostrand Company Inc., Princeton New Jersey) 1951, p. 596.

[13] Olson, G. B. and CoHen, M., J. Less Common Metals 28 (1972) 107.

[14] ONODERA, H. and TAMURA, I., N.S.F. U.S. Japan Seminar (1979) 12.

[15] Patel, J. R. and Cohen, M., Acta Metall. 1 (1953) 531.

[16] Wollants, P., De Bonte, M. and Roos, J. R., Z. Metallk. 70 (1979) 113, 146, 298.
[17] Gautier, E., Thèse de Doctorat ès-Sciences Physiques, Institut National Polytechnique de Lorraine, Nancy, 1985.

[18] Hornbogen, E., Acta Metall. 33 (1985) 595.

[19] Tokizane, M., Sup. Trans. J.I.M. 17 (1976) 345.

[20] Tokizane, M. and Maruyama, Y., Scripta Metall. 12 (1978) 233.

[21] NAKASA, K. and NaKamura, T., Scripta Metall. 12 (1978) 503.

[22] Tokizane, M., Scripta Metall. 10 (1976) 459.

[23] Tamura, I., Met. Sci. J. 16 (1982) 245.

[24] ONODERA, H. and TAMURA, I., N.S.F. U.S./Japan Seminar (1979) 12.

[25] Onodera, H., Goto, H. and Tamura, I., Sup. Trans. J.I.M. 17 (1976) 337.

[26] Onodera, H. and Tamura, I., Proceedings of the International Conference on Martensitic Transformation Cambridge Massachussets (1979) 337.

[27] Lecroisey, F. and Pineau, A., Metall. Trans. 3 (1972) 387.

[28] Gautier, E., Simon, A. and Beck, G., Proceedings of the International Conference Martensitic Transformation, The Japan Institute of Metals (1986) 503.

[29] Кот, R. A. and Weiss, V., Metall. Trans. 1 (1970) 2685.

[30] Pradhan, R. and Ansell, G. S., Metall. Trans. A 9A (1978) 793.

[31] Gautier, E., Simon, A. and Beck, G., Proceedings of Internal Conference on Strength of Metals and Alloys, edited by Mc Queen et al. (Pergamon Press) 1985 , p. 569.

[32] Gautier, E., Simon, A. and Beck, G., Acta Metall. 35 (1987) 1367. 